Check for updates

Cite this: J. Mater. Chem. B, 2022, 10,373

Received 4th June 2021

Accepted 14th December 2021

DOI: $10.1039 / \mathrm{d} 1 \mathrm{tb} 01266 \mathrm{e}$

rsc.li/materials-b

\section{Tuneable synthetic reduced graphene oxide scaffolds elicit high levels of three-dimensional glioblastoma interconnectivity in vitro $\dagger$}

\author{
Christopher J. Brown, (D) ${ }^{a}$ Thomas Simon, (D) ${ }^{b}$ Chiara Cilibrasi, (D) \\ Peter J. Lynch, (D) ${ }^{a}$ Rhiannon W. Harries, (D) ${ }^{a}$ Aline Amorim Graf, (D) a \\ Matthew J. Large, (DD a Sean P. Ogilvie, (D) a Jonathan P. Salvage, (D) d \\ Alan B. Dalton, (D) ${ }^{a}$ Georgios Giamas (iD ${ }^{c}$ and Alice A. K. King (iD) *a
}

\begin{abstract}
Three-dimensional tissue scaffolds have utilised nanomaterials to great effect over the last decade. In particular, scaffold design has evolved to consider mechanical structure, morphology, chemistry, electrical properties, and of course biocompatibility - all vital to the performance of the scaffold and how successful they are in developing cell cultures. We have developed an entirely synthetic and tuneable three-dimensional scaffold of reduced graphene oxide ( $\mathrm{rGO}$ ) that shows good biocompatibility, and favourable mechanical properties as well as reasonable electrical conductivity. Importantly, the synthesis is scaleable and suitable for producing scaffolds of any desired geometry and size, and we observe a high level of biocompatibility and cell proliferation for multiple cell lines. In particular, one of the most devastating forms of malignant brain cancer, glioblastoma (GBM), grows especially well on our rGO scaffold in vitro, and without the addition of response-specific growth factors. We have observed that our scaffold elicits spontaneous formation of a high degree of intercellular connections across the GBM culture. This phenomenon is not well documented in vitro and nothing similar has been observed in synthetic scaffolds without the use of response-specific growth factors - which risk obscuring any potential phenotypic behaviour of the cells. The use of scaffolds like ours, which are not subject to the limitations of existing two-dimensional substrate technologies, provide an excellent system for further investigation into the mechanisms behind the rapid proliferation and success of cancers like GBM. These synthetic scaffolds can advance our understanding of these malignancies in the pursuit of improved theranostics against them.
\end{abstract}

\section{Introduction}

Nanomaterial technologies have been developed in many morphologies including two-dimensional films, liquid cells and hydrogels, and three-dimensional aerogels and foams. Foam technologies have found applications in energy generation and storage devices, ${ }^{1}$ sensing and wearable electronics, ${ }^{2}$ filtration and storage ${ }^{3}$ of gases and ions, and within biomedical research ${ }^{4}$

\footnotetext{
${ }^{a}$ Department of Physics and Astronomy, University of Sussex, Brighton, BN1 9QH, UK. E-mail: Alice.King@sussex.ac.uk

${ }^{b}$ Department of Translational Genomics, Keck School of Medicine, University of Southern California, Los Angeles, CA, USA

${ }^{c}$ Department of Biochemistry and Biomedicine, University of Sussex, Brighton, BN1 9QG, UK

${ }^{d}$ School of Pharmacy and Biomolecular Sciences, University of Brighton, BN2 4GJ, UK

$\dagger$ Electronic supplementary information (ESI) available. See DOI: 10.1039/ d1tb01266e
}

and tissue engineering. ${ }^{5}$ Indeed, throughout the last decade, nanomaterials have been increasingly utilised in biological and medical applications; ${ }^{6}$ finding uses in diagnostic procedures, ${ }^{7}$ as vectors for drug delivery ${ }^{8}$ through mechanisms such as internalisation, ${ }^{9}$ as scaffolds and substrates ${ }^{10}$ for tissue engineering and regenerative medicine, ${ }^{11}$ and for the modelling of diseases such as Alzheimer's. ${ }^{12}$ The novel and diverse chemical, mechanical, and electronic properties of such materials offer a vast palette of avenues for exploration within biomedical research. ${ }^{13}$ Of these, graphene and its derivatives such as graphene oxide (GO), are particularly attractive options. ${ }^{14}$ Carbon-based nanomaterials tend to offer good biocompatibility, and well-documented chemistries that are open to a range of potentially helpful functionalisations. ${ }^{15}$ Graphene, whilst broadly applicable and well characterisable ${ }^{16}$ is experimentally difficult and expensive to both manufacture and process in industrially useful quantities, often requiring harsh solvents and surfactants to do so. While the use of graphene technologies in biomedical applications has been relatively well 
investigated, ${ }^{17}$ there remains a complex and expansive landscape of potential applications, with much uncharted territory to explore.

A less thoroughly investigated member of the graphene derivatives, GO is particularly suited to such research as it is well understood chemically, ${ }^{18}$ it offers the opportunity for simple, scaleable solution processing, and it can be easily reduced into reduced graphene oxide (rGO). The reduction process partially recovers many of the desirable properties of graphene such as high conductivity and hydrophobicity. ${ }^{19}$ For biomedical applications, this also allows for aqueous processing pre-reduction, and the reduction step then prevents dissolution of the scaffold in cell media - therefore rGO represents a viable alternative to graphene that is both much easier and much cheaper to manufacture and process.

Glioblastoma (GBM) is the most common form of malignant brain tumour, and is known to be particularly aggressive. ${ }^{20}$ One of the most pertinent reasons for the belligerence of GBM is how efficiently the cancer is able to avoid even the most intensive conventional combination treatments. ${ }^{21}$ GBM tumours are self-supporting; by a number of means including angiogenesis and vascular co-option, they grow as highly vascularised masses which can mimic and remodel existing vasculature to readily support the rapid proliferation of the cancer. $^{22,23}$ One of the most intriguing routes that GBM takes to vascularise uses the transdifferentiation of glioblastoma stem-like cells (GSCs) into endothelial cells. The mechanisms by which this transdifferentiation occur are elusive; they are particularly hard to observe naturally, and are therefore not very well documented. The process can be forced to occur in vitro via the use of targeted external signals and growth factors, ${ }^{24,25}$ but these observations are not necessarily representative of the processes as they occur in vivo, nor do they give insight into the phenotypic behaviour of the cells without external drivers. Previous studies have shown strong evidence for the differentiation and transdifferentiation of GSCs for vascularisation ${ }^{26-28}$ but were unable to entirely remove the possibility of vascular co-option and growth factor-driven changes, wherein growth factors specifically encouraged endothelial cell fates.

In this work, we have developed a tuneable, porous, threedimensional rGO foam via a simple and scaleable lyophilisation process, followed by thermal reduction (Fig. 1) - allowing us to develop a favourable tissue scaffold whilst avoiding both the challenges that accompany the processing of pristine graphene, and the limitations of two-dimensional substrates. ${ }^{29,30}$ By carefully modifying steps throughout the method, our process offers multiple opportunities to control the resultant foam properties. Considered changes to the procedure at either the precursor dispersion or reduction stages, or to the initial freezing conditions (and thereby altering the crystallite formation), can yield controllable effects upon any of foam chemistry, density, pore size distribution, pore alignment, ${ }^{31}$ mechanical stiffness, electrical conductivity, and foam solubility. ${ }^{32}$ We have used our rGO foam as a macrostructured porous scaffold on which to successfully culture GBM cells from multiple GBM cell lines as monocultures in standard cell media, without the use of any additional growth factors that would target specific cell responses. Lack of such

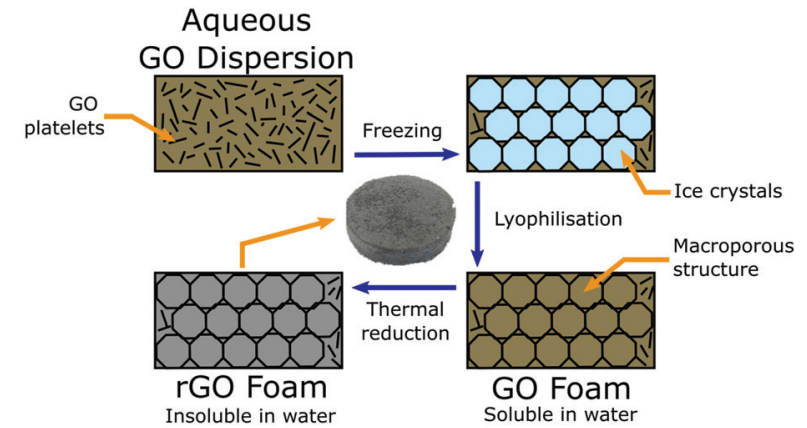

Fig. 1 Schematic detailing the synthesis of our rGO foam. Synthesis starts with an aqueous colloidal dispersion, which is cast into a mould, and then sequentially frozen, lyophilised, and thermally reduced into a macroporous rGO foam that is insoluble in water.

growth factors demonstrates that the observed cell development is not driven by external interactions the surrounding tissue, but only from any inherent phenotypic behaviour of the GBM cells or GSCs.

\section{Experimental}

\section{Materials \& synthesis of rGo foams}

'Highly concentrated' GO was purchased from Graphenea, as a gel at $2.5 \mathrm{wt} \%$, with $>95 \%$ monolayer content as measured at $0.05 \mathrm{wt} \%$.

An aqueous colloidal dispersion of GO was diluted with de-ionised (DI) water into a dispersion of the desired wt\%. DI water was used to introduce as few impurities and ionised particulates into the foams as possible. Impurities in the precursor dispersion, which could be introduced by using non-DI water, could impact both the mechanical and electrical properties of the resulting foam scaffolds as well as providing the potential for toxicity to the cells under study. The diluted precursor dispersion was then cast into a mould of desired geometry, frozen in a conventional freezer at approximately $-18.5{ }^{\circ} \mathrm{C}$, and lyophilised with a Labconco 'FreeZone $2.5^{\prime}$ into a low-density foam. This mould-based liquid processing means that there is in principle no limit to the size and shape of the sample, which is beneficial when designing a scaffold for a specific application. After synthesis, the GO foams were thermally reduced into rGO for three hours at low pressure, $P \approx 1 \mathrm{mBar}$, and set temperature $T=150{ }^{\circ} \mathrm{C}$ in a Memmert vacuum oven. This procedure is detailed schematically in Fig. 1. Reduction was undertaken until no further change in conductivity was observed and the samples had recovered a metallic lustre (Fig. S1, ESI $\dagger$ ).

\section{Material characterisation}

Foam mechanical properties were tested through compression cycles using a Stable Micro Systems 'TA.XT+' texture analyser, fitted with a $5 \mathrm{~kg}$ load cell and operating with a compression test speed of $0.05 \mathrm{~mm} \mathrm{~s}^{-1}$, an automatic force trigger at $0.1 \mathrm{~g}$, and a target strain of $15 \%$. Multiple cycles of compression were completed, and associated stress, $\sigma$, versus strain, $\varepsilon$, curves were recorded. From these, it was possible to find the Young's 
modulus of each sample, $Y$, by simply taking the gradient of the initial linear part of the curve, $Y=\mathrm{d} \sigma / \mathrm{d} \varepsilon$.

Foam conductivity was determined with a transmission line measurement, involving collection of current-voltage curves at various distances throughout the sample to determine the sample resistance, $R$, whilst minimising the influence of contact resistances. The current-voltage curves were collected with a Textronix 'Keithley 2614B' two probe system, operating in a $-20-20 \mathrm{~V}$ range with $0.02 \mathrm{~V}$ steps between measurements. The resistances were then combined with measurements of the foam geometry in order to determine the conductivity, $\sigma_{\mathrm{DC}}$, where $\sigma_{\mathrm{DC}}=1 / R_{\mathrm{s}} t$, with $R_{\mathrm{S}}$ the sample sheet resistance, computed from the gradient of a plot of $R$ against $l / w$, with $l$ the distance between measurement lines, $w$ the width of the transmission line itself, and $t$ the thickness of the foam sample.

\section{Control of foam properties}

The full foam synthesis method provides many opportunities to alter various foam properties in a controlled manner, within the limits of the foam being self-supporting. By adjusting aspects of the precursor dispersion such as the weightpercent solids content or by the addition of particular additives or dilutants, it is possible to reliably control foam chemistry, density and mechanical stiffness. Further changes can be made at the initial freezing stage - the rate of freezing and the direction of the temperature gradient in the freezing environment can affect both the size distribution of pores in the foam, as well as the general pore shape and alignment. ${ }^{31}$ In turn, these factors affect the overall mechanical properties of the foam, and how it would interact with cells as a scaffold. Finally, the reduction parameters can be adjusted to affect foam properties. ${ }^{19}$ By choosing whether to reduce the foam fully or only partially, it is possible to control the electrical conductivity and the foam's solubility in water, again changing the foam's performance as a cell scaffold.

In this study, the focus was on controlling the foam stiffness by adjusting the solids content of the precursor GO dispersion to a balance of structural integrity while still allowing sufficient cell growth and motility. A reliable balance of this was found at $2.00 \mathrm{wt} \%$ precursor solids content.

\section{Cell cultures}

U87 and LN18 GBM cells (ATCC) were maintained in minimum essential medium (MEM, Sigma-Aldrich) and Dulbecco's modified eagle medium (DMEM, Sigma-Aldrich) respectively. Cell line culture medium was supplemented with 100 units $\mathrm{mL}^{-1}$ penicillin, $100 \mu \mathrm{g} \mathrm{mL}^{-1}$ streptomycin, $2 \mathrm{mM}$ L-glutamine (PSG, Sigma-Aldrich) and $10 \%$ heat inactivated fetal bovine serum (FBS, First Link). GS090 cells (GBM patient-derived stem cells) were a kind gift from Dr David Nathanson, University of California, Los Angeles. GBM stem cells were isolated from GBM tumor samples following local Ethical Board approval. The GBM stem cells were maintained as neurospheres in (DMEM/F-12, Sigma-Aldrich) supplemented with B-27 without Vitamin A (Life Technologies), Hu EGF $\left(20 \mu \mathrm{g} \mathrm{mL}^{-1}\right)$, Hu FGF-b $\left(8 \mu \mathrm{g} \mathrm{mL} L^{-1}\right)$, Heparin $\left(2 \mathrm{mg} \mathrm{mL}^{-1}\right), 100$ units $\mathrm{mL}^{-1}$ penicillin, $100 \mu \mathrm{g} \mathrm{mL}^{-1}$ streptomycin, and Glutamax (Invitrogen). HUVEC
(ATCC) were maintained in F-12K medium (F-12K, SigmaAldrich) supplemented with 100 units $\mathrm{mL}^{-1}$ penicillin, $100 \mu \mathrm{g} \mathrm{mL}^{-1}$ streptomycin, $0.1 \mathrm{mg} \mathrm{mL}^{-1}$ heparin (Sigma-Aldrich), $30 \mu \mathrm{g} \mathrm{mL} \mathrm{m}^{-1}$ endothelial cell growth supplement (Fisher Scientific) and $10 \%$ heat inactivated fetal bovine serum (FBS, First Link).

\section{Cell studies - rGO foam scaffolds}

Cells were incubated at $37{ }^{\circ} \mathrm{C}$ in a humidified atmosphere at $5 \% \mathrm{CO}_{2}$. Cells were seeded in 6-well plates containing $2.00 \mathrm{wt} \%$ rGO foams and grown for 21 days before being fixed using $4 \%$ paraformaldehyde. Medium was changed twice a week. Foams were sterilised with UV-Ozone treatment for 30 minutes in a PSD Series Digital UV Ozone System fitted with an Ozone Elimination System (Novascan) prior to cell culture.

\section{Cell cultures - cell counts and viability}

All cell count experiments were performed on either blank PET slips, or PET slips with a $200 \mu \mathrm{m}$ rod-coated rGO film. For the rGO films, GO at $2.00 \mathrm{wt} \%$ was cast onto blank PET, rod coated to $200 \mu \mathrm{m}$ thickness, air dried, and then thermally reduced into rGO for three hours at low pressure, $P \approx 1 \mathrm{mBar}$, and set temperature $T=150{ }^{\circ} \mathrm{C}$ in a vacuum oven (Memmert). Both PET and $\mathrm{rGO} / \mathrm{PET}$ slips were sterilised with UV-Ozone treatment for 30 minutes in a PSD Series Digital UV Ozone System fitted with an Ozone Elimination System (Novascan) prior to cell culture.

For the Trypan blue dye exclusion assay, 20000 cells well $^{-1}$ were cultured in a 24-well plate in the presence of either blank PET or PET and rGO. After 3 and 5 days, cells were stained using the Trypan blue dye (ThermoFisher) to count cell numbers and determine the viability. The results reported are the mean of three biological replicates.

For the Cell Titer Glo measurement, 3000 cells well $^{-1}$ were cultured in a 96-well plate in the presence of either blank PET or PET and rGO. After 3 and 5 days, $100 \mu \mathrm{L}$ of Cell Titer Glo reagent (Promega) were added to each well and luminescence was recorded with a plate reader (Promega GloMax Multi Detection System). The results reported are the mean of three biological replicates.

For the crystal violet measurement, 3000 cells well $^{-1}$ were cultured in a 96-well plate in the presence of either blank PET or PET and rGO. After 3 and 5 days, cells were fixed with $4 \%$ PFA for 15 minutes. Plates were then washed 3 times with PBS. Cells were stained with $100 \mu \mathrm{L}$ of $0.1 \%$ crystal violet (as weight per unit volume of solution) in 10\% ethanol for 20 minutes and plates were washed 3 times in PBS and left to air dry overnight. The day after, the bound dye was solubilized by adding $100 \mu \mathrm{L}$ of $10 \%$ acetic acid. The absorbance at $590 \mathrm{~nm}$ was recorded with a plate reader (Promega GloMax Multi Detection System). The results reported are the mean of three biological replicates.

\section{Scanning electron microscopy}

A Zeiss SIGMA field emission gun scanning electron microscope (SEM) with Zeiss in-lens secondary electron detector was operated with a $1.00 \mathrm{kV}$ electron high tension, and with typical working distances in the region of $3 \mathrm{~mm}$. The rGO foam samples themselves have an innate conductivity so no further 
sample preparation was necessary prior to the electron microscopy, other than mounting the foam onto SEM sample stubs with carbon tape. To mount the samples, the foam was cut and attached to SEM stubs in multiple different orientations with carbon tape to image the sample from multiple directions, including cross-sections. To help clarify SEM images of cell culture samples, they were false coloured in blue to highlight any cell-derived structures.

\section{Determination of average pore diameter}

From SEM images, an estimate of average foam pore size was calculated by taking two measurements of pore diameters in opposing directions for each identifiable pore in an image. The numerical average of these two measurements was computed, as well as an associated uncertainty, which was estimated as half the difference of the two measurements for each pore. These were combined into a weighted mean, $\overline{D_{p}}$, as well as a statistical standard error on the weighted mean, $\sigma_{\overline{D_{p}}}$, where

$$
\overline{D_{p}}=\frac{\sum_{i} w_{i} D_{p_{i}}}{\sum_{i} w_{i}} \text {, and } \sigma_{\overline{D_{p}}}=\sigma_{D_{p}} \cdot \sqrt{\sum_{i} w_{i}^{\prime 2}},
$$

in which $D_{p_{i}}$ are the individual pore diameter measurements, $w_{i}$ and $w_{i}^{\prime}$ are the standard and normalised statistical weights on each measurement respectively, and $\sigma_{D_{p}}$ is the standard deviation of the measured pore diameters.

\section{Fluorescence microscopy}

An Olympus 'BX53M' optical microscope fitted with a 'U-MIXR' fluorescence attachment was used to image samples in both standard optical and fluorescent regimes, using both bright-field and dark-field modes and varying magnifications as appropriate.

For fluorescence imaging, cells were stained to highlight the expression of the CD34 protein. Cells were fixed with a $4 \%$ PFA prior to permeabilisation. Cell staining was undertaken by preparing a $1 \%$ permeabilising solution of mild Triton-X 100 in phosphate-buffered saline (PBS); a 10\% blocking solution of heat-inactivated normal goat serum blocking reagent (Alexa Fluor, Invitrogen) in PBS; a 1:1000 solution of mouse IgG1 CD34 monoclonal antibody (Thermo Fisher Scientific) in a $1 \%$ blocking solution in PBS; and a 1:1000 solution of $488 \mathrm{~nm}$ goat anti-mouse fluorophore (Alexa Fluor, Sigma-Aldrich) in PBS. Then, by the conventional sequential washing of the samples with permeabilising solution, blocking solution, CD34 antibody solution, and fluorophore solution, and respective incubations of the sample to allow the various solutions to take effect, the samples were stained to highlight the expression of CD34.

\section{Results and discussion}

Two particularly useful aspects of GO as a scaffold material are that it is easy to synthesise, ${ }^{33}$ so it is readily commercially available, and after its initial synthesis it is easy and environmentally friendly to process as an aqueous solution without the need for any additives or surfactants. The fact that GO can be liquid-processed allows for the precursor dispersion to be moulded to any desired scaffold geometry before lyophilising into a dry GO foam. Since the shape is dictated only by the choice of mould before freezing, any number of different shapes are theoretically possible, making it easy to ensure morphological compatibility with real tissues. In addition, the size of the scaffold is restricted only by the size of the freeze dryer; with an industrial freeze dryer, it would in principle be possible to make large scaffolds. Conveniently, the inherent solubility of GO in water can be removed via simple thermal reduction into $\mathrm{rGO}$ without the need for any cytotoxic reducing agents that could result in a less biocompatible scaffold. This reduction step leaves a non-cytotoxic scaffold of easily engineered size and shape which maintains its integrity in water, making it robust to biological studies that require aqueous cell media. The reduction step also recovers some electrical conductivity within the scaffold; the foam conductivity increased from around $10^{-4} \mathrm{~S} \mathrm{~m}^{-1}$ as GO up to conductivities of order $10^{-1} \mathrm{~S} \mathrm{~m}^{-1}$ as rGO (Fig. S1, ESI $\dagger$ ). The electrical conductivity is beneficial as it enables SEM imaging without the need for any further treatment or coating, and it is thought that it may aid the growth of cells that expect a conductive microenvironment such as those within the central nervous system. ${ }^{34,35}$ There is also the potential for electrical stimulation of cell colonies, ${ }^{4,36,37}$ though this is not investigated in this work.

The mechanical stiffness of the foam scaffold is an important parameter, as stiffness is one of the foremost external cues that can dictate cell development, particularly in the case of stem cells. $^{38}$ Cells are known to change their behaviour to preferentially develop along certain pathways when grown in conditions which offer specific microenvironments that mimic those that the cells would encounter in vivo. ${ }^{39,40}$ For a tissue scaffold to grow GBM cells successfully, it must offer a mechanical stiffness close to that of brain tissue. ${ }^{41}$ Ideally, one would be able to tailor the properties of a scaffold - tuning the material to suit the cells under study, and help to encourage their proliferation in a way that closely mirrors their in vivo behaviour. ${ }^{42}$ In addition, one could tune the scaffold to vary cell behaviours; the scaffold could be purpose-made to selectively encourage cell development and to promote the differentiation of stem cells along certain

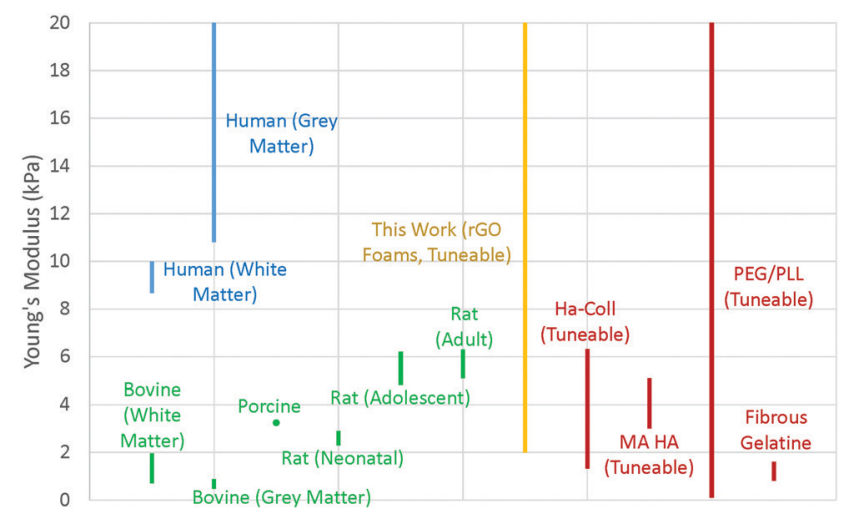

Fig. 2 Mechanical properties for brain tissues of different species, and of other tissue scaffolds designed for brain cell research, as reported in literature (Table S2, ESI $\dagger$ ). Where the modulus was not explicitly provided, these values were calculated by computing the ratio of stress to strain in the linear region of the data values as quoted in the literature. 

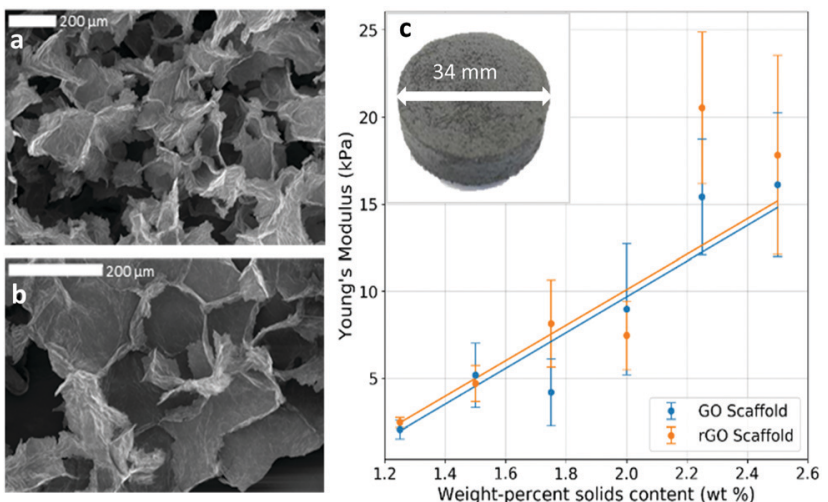

Fig. 3 Physical properties of GO foam and rGO foam scaffold. (a) and (b) SEM images showing the typical porous structure of the dry 2.00 wt\% foam. (c) Scaffold Young's modulus increases linearly with solid contents in the foam, demonstrating control over scaffold properties. Inset is an optical photograph of a whole foam scaffold sample showing cylindrical mould geometry.

pathways, ${ }^{43}$ for example. The synthesised foams in this work were found to have low and tuneable Young's moduli, typically in the region of 2-20 kPa, (Fig. 2 and 3c), by varying the precursor dispersion solids content.

This compares favourably with other brain tissue scaffolds in the literature ${ }^{44-47}$ which show moduli generally in the range 1-20 kPa (Fig. 2), and is significantly different to, for example, scaffolds for vascular tissue engineering which have Young's modulus of order $\mathrm{MPa}^{48}$ Brain tissue is known to have a Young's modulus that varies with all of region, ${ }^{49}$ matter type,$^{50}$ age, ${ }^{51}$ myelin content, ${ }^{52}$ and species ${ }^{44,50,52,53}$ but is typically in the region of $10-20 \mathrm{kPa}$ in humans (Fig. 2), so our rGO scaffold is a promising candidate for studies of cells that would expect such tissues, such as GBM.

It has been shown that different scaffold morphologies give rise to a range of cell-scaffold effects. ${ }^{54}$ In particular for porous scaffold technologies, pore size plays an important role in cell seeding efficiency, ${ }^{55}$ cell attachment and proliferation, ${ }^{56}$ and overall cell function. ${ }^{57}$ From SEM imaging, it was observed that our rGO foams also had a relatively uniform, macroporous structure (Fig. 3a and b), with average pore diameters of (199 \pm 13$) \mu \mathrm{m}$. Morphologically, this compares to other
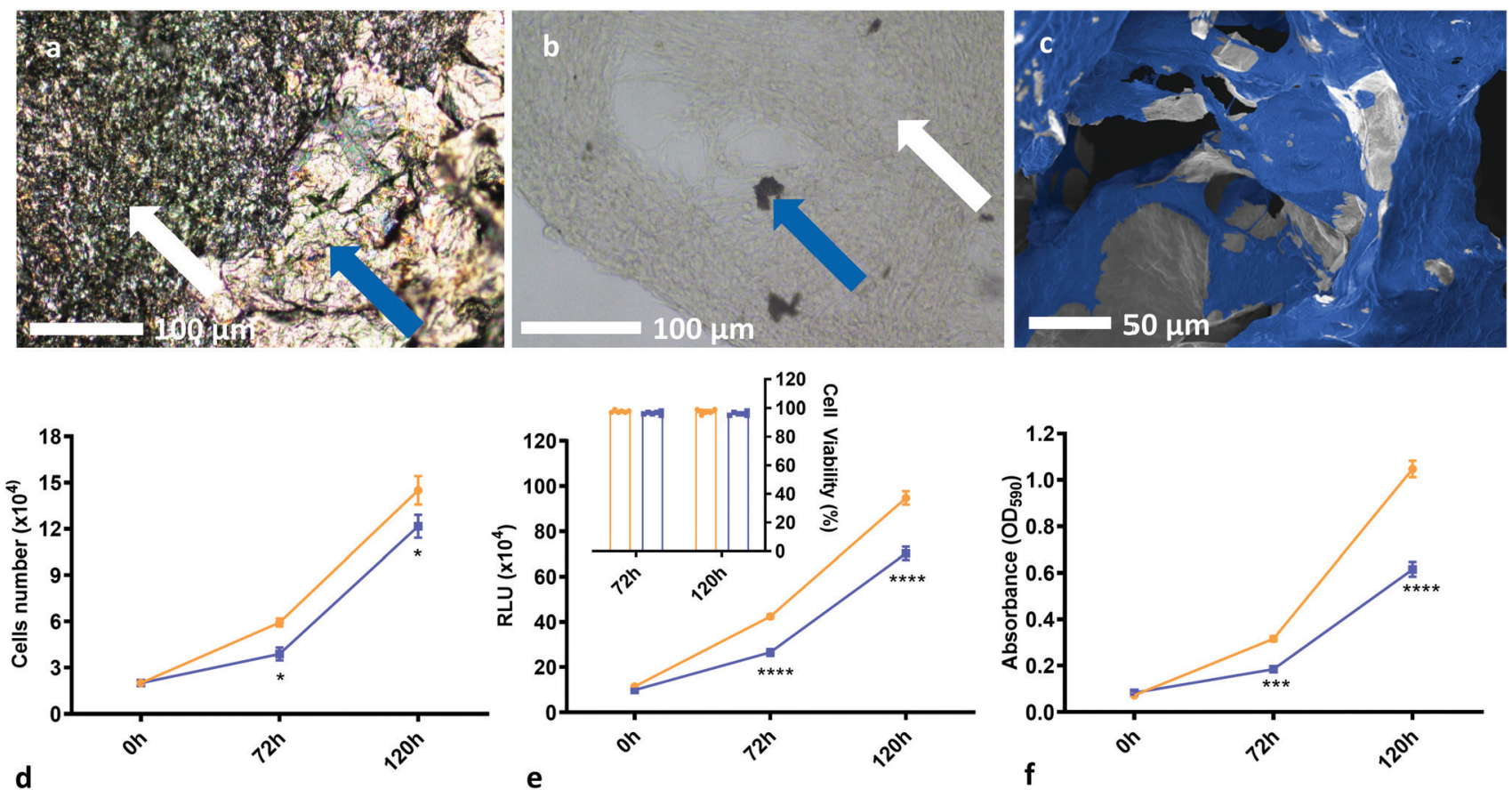

Fig. 4 Demonstration of biocompatibility and the suitability of $r G O$ foams as tissue scaffolds. (a) Optical micrographs of masses of GBM cells grown on our rGO foam scaffolds, with white arrows showing cells, and blue arrows demarking the rGO scaffold. (b) Optical micrograph of a large mass of GBM cells grown on the foam, with most of the foam having been broken up and removed from the sample, with white arrows showing cells, and blue arrows again demarking the rGO scaffold. (c) SEM image of U87 cells grown on the rGO foam scaffold. This has been false coloured for clarity, and shows excellent cell coverage on the foams across multiple cell lines. (d) Plot of cell count over time comparing a blank PET control substrate to an rGO/PET substrate, both in a 24-well plate. Results reported are the mean of three biological replicates. Cell viability was consistently measured between $95 \%$ and $100 \%$ for both conditions across all biological replicates. (e) Plot of cell luminescence in the presence of Cell Titer Glo reagent, comparing a blank PET control substrate to an $\mathrm{rGO} / \mathrm{PET}$ substrate, both in a 96-well plate. Results reported are the mean of three biological replicates. From the cell count measurements, cell viability was computed, and these results are shown inset. (f) Plot of cell absorbance at $590 \mathrm{~nm}$, with cells stained with $0.1 \%$ crystal violet solution, comparing a blank PET control substrate to an $\mathrm{rGO} / \mathrm{PET}$ substrate, both in a 96 -well plate. Results reported are the mean of three biological replicates. (d)-(f) suggest that the rGO chemistry is suitably biocompatible; we see no cytotoxicity and good cell viability, albeit with a somewhat decreased growth rate. The asterisk notation denotes that these measurements have certain statistical $p$-values, as calculated from two-way ANOVA tests with Šíák's multiple comparisons tests. Statistical probabilities of $p \leq 0.05,0.001$, and 0.0001 are demarked as *, ${ }^{* * *}$, and ${ }^{* * * *}$ respectively. SEM images without additional false colouring, as well as some additional SEM from GS090 and HUVEC lines, and optical images are provided for reference in Fig. S3 and S4i (ESI $\uparrow$ ). 
graphene foam scaffold technologies for brain tissue engineering, in which strong cell adhesion and mass transport were observed. ${ }^{58}$ This suggests that our scaffold geometry presents a good option for tissue scaffolds, but does not rely on any complex or potentially toxic processing techniques after the initial GO synthesis.

Moreover, the weight-percentage of solids content of the precursor dispersion affects the strut diameter and wall thickness of the resulting foam. When a low weight-percentage has been used, the foam structure is observed to be unstable and to collapse easily. This provides a lower limit for the foam density, and the initial weight-percentage of solids content of the precursor dispersion should be chosen accordingly. While an upper limit to the density has not been observed, it is postulated that if too high an initial weight-percentage is used, then it will lead to significantly denser foams; the average strut diameter and wall thicknesses should increase, reducing both porosity and pore interconnectivity and hence limiting the mass transport needed for sufficient cell growth. This would inhibit cell motility and proliferation throughout the structure. For optimal scaffold properties, a trade-off must be found between loose, porous foams for optimal cell movement and robust mechanical properties such as stiffness for structural integrity. In this work, $2.00 \mathrm{wt} \%$ foams with dry densities of around $20 \mathrm{mg} \mathrm{cm}{ }^{-3}$ have proven to be sufficiently biocompatible scaffolds which allow good cell proliferation over the whole structure (Fig. $4 \mathrm{a}-\mathrm{c}$ ). This is observed for different cell lines and several runs over different scaffold densities, indicating good repeatability and biocompatibility over a range of mechanical and morphological properties.

The rGO chemistry itself is shown to be suitably biocompatible as shown by cell count, luminosity and absorbance data (Fig. 4d-f). Two-way ANOVA tests, with Š́dák's multiple comparisons tests, of the cell proliferation data show that there is a statistical difference in cell number when comparing cells in the presence of rGO to the control, with $P \leq 0.05$ for both conditions. However, cell viability was greater than $95 \%$ for all conditions (Fig. 4e inset), suggesting that the presence of rGO does not significantly prevent the cells from being a sufficiently viable, metabolically active biomass and confirming that the rGO chemistry is not cytotoxic to the cells, but may somewhat slow their proliferation rate. This decrease in growth rate may be linked to the differentiation process, whereby metabolic changes cause proliferation to slow in order to enable differentiation $^{59}$ - but further studies are required to confirm this. It is important to note that the cell count experiments were undertaken using thick films of rGO on a PET substrate as opposed to samples of the rGO foam scaffold. This is because isolating the cells from the foam to count them after culture is not possible - the cells become too ingrained within the porous structure that they cannot be removed to provide a reliable count. From the data obtained on the rGO films, we have shown good chemical biocompatibility, and from SEM images
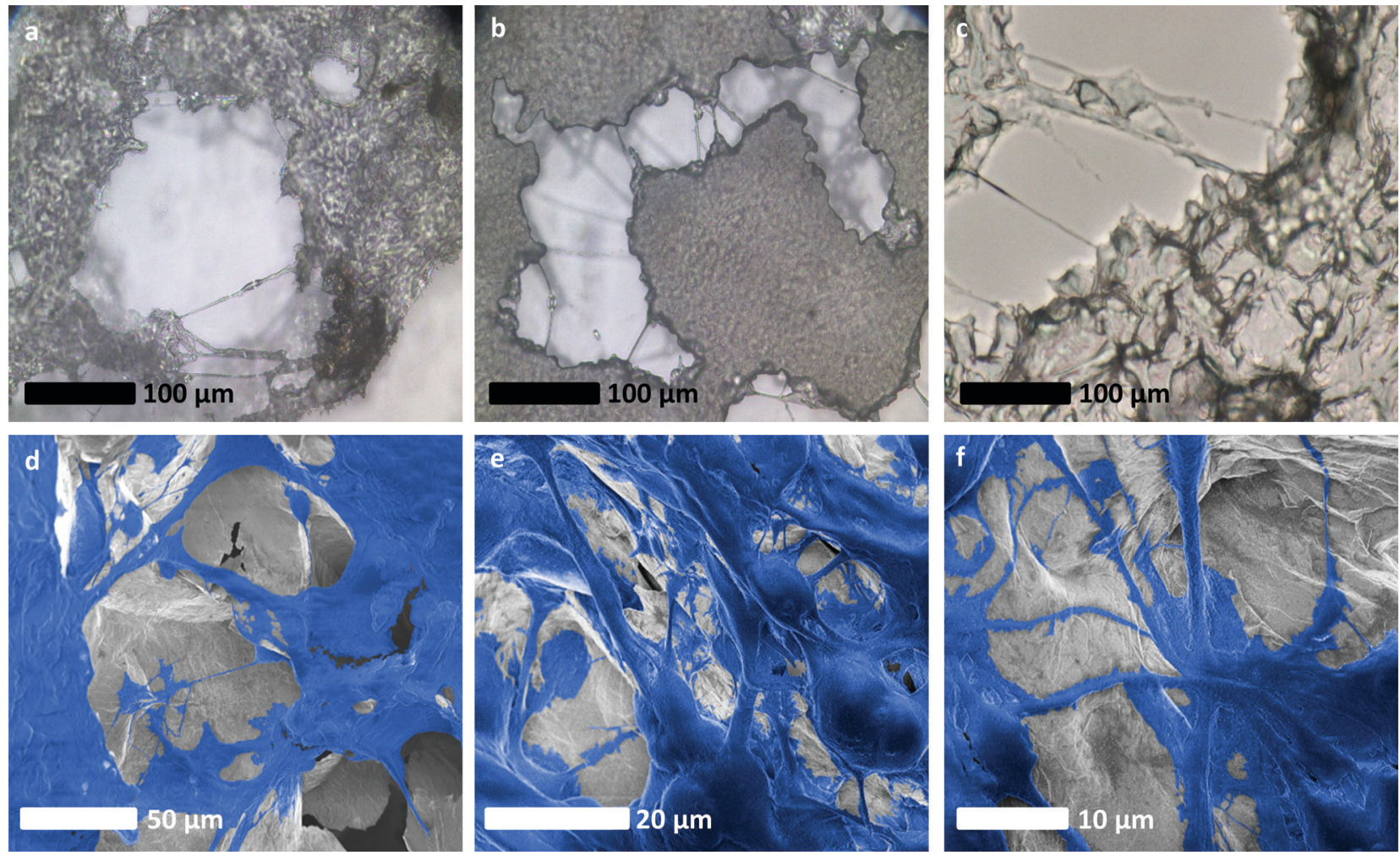

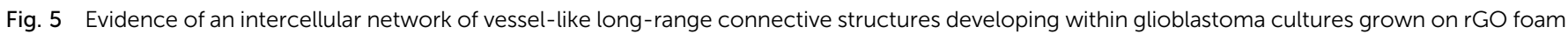

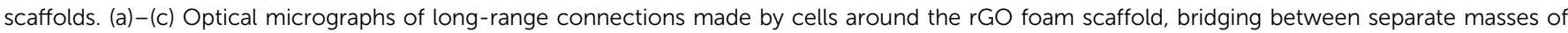

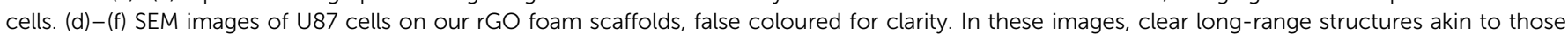
associated with vascularisation are evident. SEM images without additional false colouring are provided for reference in Fig. S4ii (ESI $\dagger$ ). 
of the cells on the foam scaffolds we see that the porous foam morphology does not inhibit cell growth or motility - and we therefore conclude that the rGO foams are inherently biocompatible, and viable cell scaffolds.

Interestingly, long-range cell connections and high levels of colony interactions are observed throughout the entire structure, possibly due to the three-dimensional nature of the scaffold, as connections need to be maintained across dimensions (Fig. 5).
The high level of porosity and connectivity within the scaffold implies that it enables successful cell interaction on a multidimensional level, where the cells can communicate ${ }^{60}$ throughout the whole structure and are not limited to surface signalling; an inherent limitation of two-dimensional technologies. This possibly results in a suitable environment for the formation of vessel-like structures, that may, in part, be made up of microtubules or even tunnelling nanotubes ${ }^{61}$ which would aid the
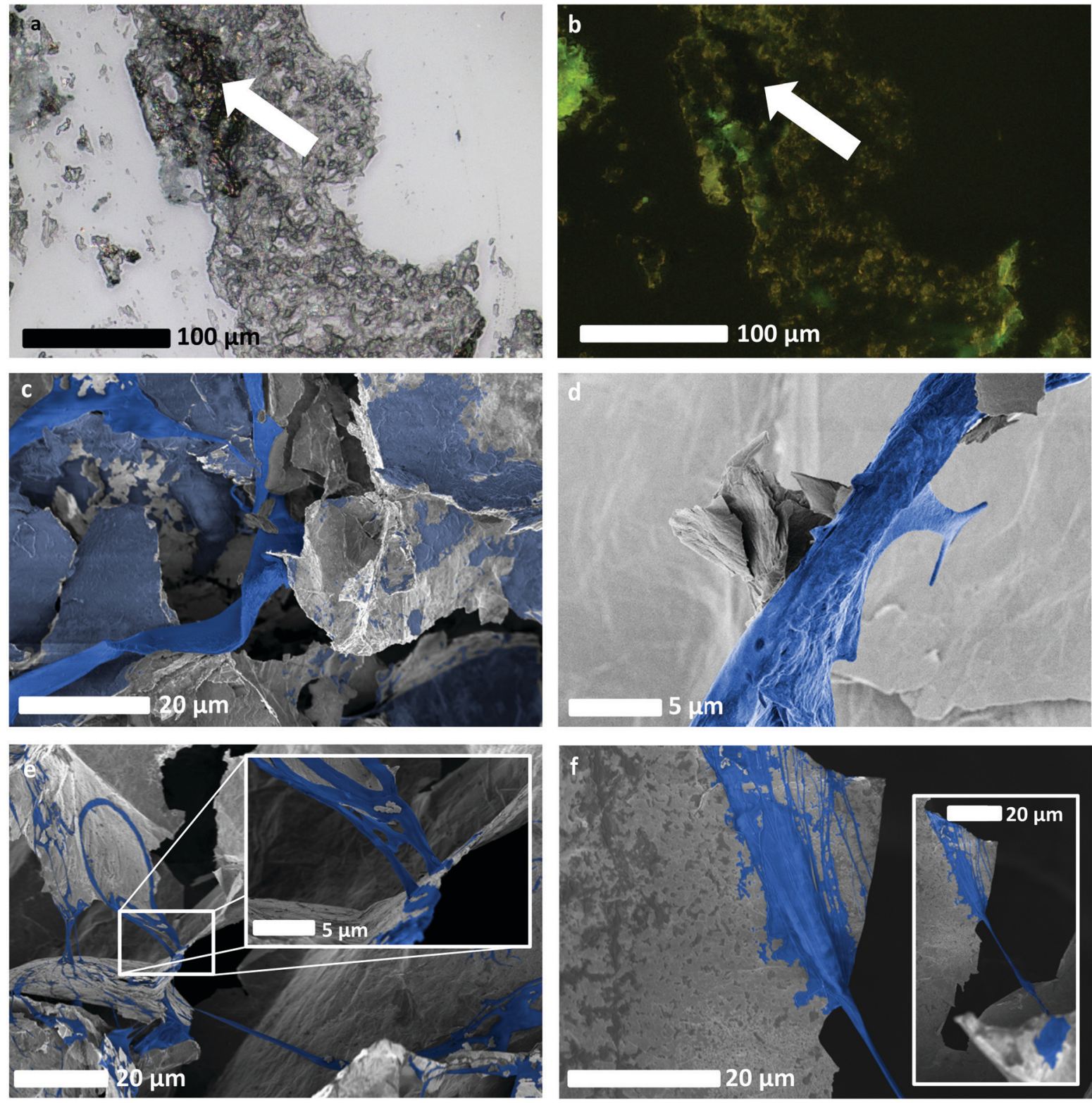

Fig. 6 Evidence of spontaneous neovascularisation of GBM on rGO foam scaffolds, without the use of targeted growth factors beyond fetal bovine serum. (a) and (b) Optical, and fluorescence imaging respectively, of GBM cells that had been stained to highlight expression of CD34, typically exhibited by endothelial cells. These show cell fluorescence and scaffold non-fluorescence, and hence presence and distribution of cells as an indicator of healthy cell proliferation, as well as potentially evidencing the change of phenotype of GBM cells. (c) SEM image of a long-range connective structure formed spontaneously by HUVEC cells, as well as other HUVEC cells that have grown upon the scaffold. (d) SEM image that appears to show a feature resembling a sprout emerging from another spontaneously-formed HUVEC-derived vessel-like structure. Sprouting is indicative of neovascularisation and angiogenesis. (e) SEM image showing a network of long, connected vessel-like structures formed from GBM cells from the LN18 cell line. The inset shows a zoomed-in view of the LN18 cell culture, highlighting what appears to be evidence of intussusception and therefore a potential change of phenotype in the LN18 cells. (f) SEM image showing the connection point of an LN18 vessel-like structure to the rGO scaffold, with a zoomed-out view showing longer vessel-like structure inset. SEM images without additional false colouring are provided for reference in Fig. S4iii (ESI $†$ ). 
growth of a tumour mass. This vessel-like connection formation is not well observed ${ }^{62}$ in literature, and the precise role of microtubules in cancer is not well understood; ${ }^{63}$ their potential presence in our cultures may indicate that the rGO scaffold is an accurate facsimile of in vivo tissues, and it may also be typical of how successful GBM is in forming a connected tumour cell mass. This in vitro analogue could therefore present a new opportunity to study how tumour masses develop in the body which is cheaper and less technically challenging than previous technologies, and could potentially reduce the need for animal models. Additionally, whilst many drugs target vascularisation and microtubule formation, ${ }^{64}$ our scaffold opens up the possibility of further testing and developing drugs that target these phenomena by testing on accurate synthetic in vitro analogues.

Fluorescence microscopy of stained U87 cells (Fig. 6a and b) demonstrates substantial expression of CD34 proteins throughout the cell culture. The arrows therein indicate that the scaffold itself isn't stained, so the observed fluorescence is not an artefact of any absorbance of the fluorophore by the rGO scaffold. CD34 is associated with endothelial progenitor cells ${ }^{65}$ however the U87 line is not typically considered to have an endothelial phenotype. Hence, the observed expression of CD34 in the culture indicates the potential occurrence of transdifferentiation to endothelial cells - the cells from this line may have changed phenotype in order to form intercellular connections throughout the tumourlike mass. Notably from the cells cultured on our rGO foam scaffolds, spontaneous formation of vessel-like long-range connective structures is seen (Fig. 6c). Further, in the cancer cell lines we observe features that appear similar to those associated with advanced angiogenesis, such as sprouts (Fig. 6d) and intussusception - a type of angiogenesis whereby a vessel will split to form a new vascular branch ${ }^{66}$ (Fig. 6e and inset) - however, further investigation would be required to determine the nature of these structures. There are also instances of long-range connections between cell sites (Fig. 6f) which may represent intercellular communication throughout all dimensions of the scaffold.

These observations, as well as the observed expression of CD34 suggests that at least some tumour cell lines may be able to change phenotype and generate endothelial cells to support the growth of the malignancy. We observe the same structures from primary human umbilical vein endothelial cells (HUVECs) grown on our scaffold. Unlike the GBM cell lines, one would expect HUVECs to naturally form endothelial cells and the observed vessel-like structures without needing to change phenotype. With further investigation, the scaffold technology developed in this work could help to identify whether different types of tumours evade intensive combination treatments such as chemo- and radiotherapy by spontaneously changing phenotype.

\section{Conclusions}

Tailored tissue scaffolds are pivotal to the future of tissue engineering and will enable deeper understanding of diseases such as cancers. In this work, we have developed a threedimensional porous rGO foam tissue scaffold via simple lyophilisation and thermal reduction of commercially available graphene oxide. Our scaffold has favourable biocompatibility, morphology, and electrical conductivity, as well as tuneable mechanical properties and so can be optimised to suit specific cell lineages as desired. In particular, we have obtained a range of mechanical stiffnesses comparable to those of brain tissues, and as such our scaffold is well suited to cells that naturally grow upon soft tissues in vivo.

After developing cell cultures from multiple cell lines on our rGO scaffold, cell counts suggest sufficient chemical biocompatibility and good cell viability, albeit with a somewhat reduced proliferation rate. This change in proliferation may be linked to some occurrence of differentiation of the cells on our scaffold. Moreover, from GBM cells grown as monocultures, a network of long-range three-dimensional intercellular connections and tubular structures were observed throughout the entirety of the scaffold. These connections may represent a means of intercellular communication throughout the three-dimensional extent of the tumour-like mass, which cannot be observed in two-dimensional substrates. The observed tubular structures could feasibly be of vascular or tunnelling nanotube phenotypes, and early evidence suggests that there could be some form of spontaneous endothelial cell generation occurring within the GBM cell lines on our scaffold. We observe protein expression in GBM cell lines such as U87 which is usually associated with endothelial cells and not GBM. This observation presents evidence that these cells had undergone an unprovoked change of phenotype without the use of any targeted growth factors. These observations could provide new insight into the spontaneous processes involved in the rapid proliferation and plasticity of cancers such as GBM as they would occur in vivo, and therefore how robust these cancers are to even the most aggressive of intensive treatment regimes.

This work offers a new uniquely simple, synthetic and reproducible in vitro method of examining the plethora of processes involved in the behaviours that lead to rapid cancer cell proliferation and tumour success - a result that marks an improvement over existing two-dimensional technologies. Our scaffold is simple, cheap, scaleable, and does not rely on any specific external growth factors that could mask any potentially important phenotypic behaviours of the cells under study, which is a substantial benefit over some existing threedimensional technologies. Further, the scaffold is entirely synthetic and three-dimensional, providing an alternative test bed and thereby could, with further development, reduce the need for animal models. With significant further investigation, it could be used to both further our understanding of, and pave the way for the development of new theranostics against cancers such as GBM by targeting the underlying mechanisms that support their growth.

\section{Author contributions}

C. J. B. - Data curation, formal analysis, methodology, writing original draft, reviewing \& editing. T. S. - conceptualisation, formal analysis, investigation, methodology, writing - 
reviewing \& editing. C. C. - investigation, formal analysis, resources, writing - reviewing \& editing. P. J. L. - investigation, methodology, supervision, writing - reviewing \& editing. R. W. H. - formal analysis, investigation, writing - reviewing \& editing. A. A. G - investigation, writing - reviewing \& editing. M. J. L investigation, writing - reviewing \& editing. S. P. O - investigation, writing - reviewing \& editing. J. P. S. - investigation, resources, writing - reviewing \& editing. A. B. D. - conceptualisation, funding acquisition, project administration, resources, supervision, writing - reviewing \& editing. G. G. - conceptualisation, project administration, resources, supervision, writing reviewing \& editing. A. A. K. K. - conceptualisation, formal analysis, funding acquisition, investigation, methodology, project administration, resources, supervision, writing - reviewing \& editing.

\section{Conflicts of interest}

There are no conflicts to declare.

\section{Acknowledgements}

The authors are grateful to the EPSRC for funding C. J. B through a Doctoral Training Partnership, as well as the University of Sussex for providing funding from the University of Sussex Strategic Development Fund. Work in the laboratory of Prof Giamas was supported by Action Against Cancer. Further thanks are extended to Dr David Nathanson, University of California, Los Angeles for the kind provision of the GS090 cell samples.

\section{Notes and references}

1 J. Mao, J. Iocozzia, J. Huang, K. Meng, Y. Lai and Z. Lin, Graphene Aerogels for Efficient Energy Storage and Conversion, Energy Environ. Sci., 2018, 11(4), 772-799, DOI: 10.1039/C7EE03031B.

2 X. Jin, H. Jiang, G. Li, B. Fu, X. Bao, Z. Wang and Q. Hu, Stretchable, Conductive PAni-PAAm-GOCS Hydrogels with Excellent Mechanical Strength, Strain Sensitivity and Skin Affinity, Chem. Eng. J., 2020, 394, 124901, DOI: 10.1016/ j.cej.2020.124901.

3 X. Mi, G. Huang, W. Xie, W. Wang, Y. Liu and J. Gao, Preparation of Graphene Oxide Aerogel and Its Adsorption for $\mathrm{Cu}^{2+}$ Ions, Carbon, 2012, 50(13), 4856-4864, DOI: 10.1016/j.carbon.2012.06.013.

4 O. Akhavan, E. Ghaderi, S. A. Shirazian and R. Rahighi, Rolled Graphene Oxide Foams as Three-Dimensional Scaffolds for Growth of Neural Fibers Using Electrical Stimulation of Stem Cells, Carbon, 2016, 97, 71-77, DOI: 10.1016/ j.carbon.2015.06.079.

5 S. Bahrami, N. Baheiraei, M. Mohseni, M. Razavi, A. Ghaderi, B. Azizi, N. Rabiee and M. Karimi, ThreeDimensional Graphene Foam as a Conductive Scaffold for
Cardiac Tissue Engineering, J. Biomater. Appl., 2019, 34(1), 74-85, DOI: 10.1177/0885328219839037.

6 L. Zhang, Applications, Challenges and Development of Nanomaterials and Nanotechnology, J. Chem. Soc. Pak., 2020, 42(5), 658-666.

7 D. Quesada-González and A. Merkoçi, Nanomaterial-Based Devices for Point-of-Care Diagnostic Applications, Chem. Soc. Rev., 2018, 47(13), 4697-4709, DOI: 10.1039/C7CS00837F.

8 T. Shao, J. Wen, Q. Zhang, Y. Zhou, L. Liu, L. Yuwen, Y. Tian, Y. Zhang, W. Tian, Y. Su, Z. Teng, G. Lu and J. Xu, NIR Photoresponsive Drug Delivery and Synergistic ChemoPhotothermal Therapy by Monodispersed-MoS $\mathrm{M}_{2}$ Nanosheets Wrapped Periodic Mesoporous Organosilicas, J. Mater. Chem. B, 2016, 4(47), 7708-7717, DOI: 10.1039/ C6TB02724E.

9 R. W. Harries, C. J. Brown, L. Woodbine, A. Amorim Graf, M. J. Large, K. Clifford, P. J. Lynch, S. P. Ogilvie, A. B. Dalton and A. A. K. King, Cell-Substrate Interactions Lead to Internalization and Localization of Layered $\mathrm{MoS}_{2}$ Nanosheets, ACS Appl. Nano Mater., 2021, 4(2), 2002-2010, DOI: $10.1021 /$ acsanm.0c03338.

10 R. W. Harries, C. J. Brown, S. P. Ogilvie, M. J. Large, A. Amorim Graf, K. Clifford, T. Simon, G. Giamas, A. B. Dalton and A. A. K. King, Langmuir Films of Layered Nanomaterials: Edge Interactions and Cell Culture Applications, J. Phys. Chem. B, 2020, 124(33), 7184-7193, DOI: 10.1021/acs.jpcb.0c05573.

11 M. Karimi, S. Bahrami, H. Mirshekari, S. M. M. Basri, A. B. Nik, A. R. Aref, M. Akbari and M. R. Hamblin, Microfluidic Systems for Stem Cell-Based Neural Tissue Engineering, Lab Chip, 2016, 16(14), 2551-2571, DOI: 10.1039/ C6LC00489J.

12 J. Park, B. K. Lee, G. S. Jeong, J. K. Hyun, C. J. Lee and S.H. Lee, Three-Dimensional Brain-on-a-Chip with an Interstitial Level of Flow and Its Application as an in Vitro Model of Alzheimer's Disease, Lab Chip, 2015, 15(1), 141-150, DOI: 10.1039/C4LC00962B.

13 O. Salata, Applications of Nanoparticles in Biology and Medicine, J. Nanobiotechnol., 2004, 2(1), 3, DOI: 10.1186/ 1477-3155-2-3.

14 V. C. Sanchez, A. Jachak, R. H. Hurt and A. B. Kane, Biological Interactions of Graphene-Family Nanomaterials: An Interdisciplinary Review, Chem. Res. Toxicol., 2012, 25(1), 15-34, DOI: 10.1021/tx200339h.

15 S. R. Shin, Y.-C. Li, H. L. Jang, P. Khoshakhlagh, M. Akbari, A. Nasajpour, Y. S. Zhang, A. Tamayol and A. Khademhosseini, Graphene-Based Materials for Tissue Engineering, Adv. Drug Delivery Rev., 2016, 105, 255-274, DOI: 10.1016/j.addr.2016.03.007.

16 A. A. Graf, S. P. Ogilvie, H. J. Wood, C. J. Brown, M. Tripathi, A. A. K. King, A. B. Dalton and M. J. Large, Raman Metrics for Molybdenum Disulfide and Graphene Enable Statistical Mapping of Nanosheet Populations, Chem. Mater., 2020, 32(14), 6213-6221, DOI: 10.1021/acs.chemmater.0c02109.

17 C. Chung, Y.-K. Kim, D. Shin, S.-R. Ryoo, B. H. Hong and D.-H. Min, Biomedical Applications of Graphene and 
Graphene Oxide, Acc. Chem. Res., 2013, 46(10), 2211-2224, DOI: 10.1021/ar300159f.

18 A. A. K. King, B. R. Davies, N. Noorbehesht, P. Newman, T. L. Church, A. T. Harris, J. M. Razal and A. I. Minett, A New Raman Metric for the Characterisation of Graphene Oxide and Its Derivatives, Sci. Rep., 2016, 6(1), 19491, DOI: 10.1038/srep19491.

19 S. Pei and H.-M. Cheng, The Reduction of Graphene Oxide, Carbon, 2012, 50(9), 3210-3228, DOI: 10.1016/ j.carbon.2011.11.010.

20 S. Baisiwala, B. Auffinger, S. P. Caragher, J. M. Shireman, R. Ahsan, G. Lee, T. Hasan, C. Park, M. R. Saathoff, A. C. Christensen and A. U. Ahmed, Chemotherapeutic Stress Induces Transdifferentiation of Glioblastoma Cells to Endothelial Cells and Promotes Vascular Mimicry, Stem Cells Int., 2019, 2019, 1-14, DOI: 10.1155/2019/6107456.

21 Y. Soda, C. Myskiw, A. Rommel and I. M. Verma, Mechanisms of Neovascularization and Resistance to AntiAngiogenic Therapies in Glioblastoma Multiforme, J. Mol. Med., 2013, 91(4), 439-448, DOI: 10.1007/s00109-013-1019-z.

22 J. R. Kane, The Role of Brain Vasculature in Glioblastoma, Mol. Neurobiol., 2019, 56(9), 6645-6653, DOI: 10.1007/ s12035-019-1561-y.

23 J. D. Lathia, S. C. Mack, E. E. Mulkearns-Hubert, C. L. L. Valentim and J. N. Rich, Cancer Stem Cells in Glioblastoma, Genes Dev., 2015, 29(12), 1203-1217, DOI: 10.1101/gad.261982.115.

24 C. Martelli, A. King, T. Simon and G. Giamas, GrapheneInduced Transdifferentiation of Cancer Stem Cells as a Therapeutic Strategy against Glioblastoma, ACS Biomater. Sci. Eng., 2020, 6(6), 3258-3269, DOI: 10.1021/acsbiomaterials.0c00197.

25 S. El Hallani, B. Boisselier, F. Peglion, A. Rousseau, C. Colin, A. Idbaih, Y. Marie, K. Mokhtari, J. L. Thomas, A. Eichmann, J. Y. Delattre, A. J. Maniotis and M. Sanson, A New Alternative Mechanism in Glioblastoma Vascularization: Tubular Vasculogenic Mimicry, Brain, 2010, 133(4), 973-982, DOI: 10.1093/ brain/awq044.

26 L. Ricci-Vitiani, R. Pallini, M. Biffoni, M. Todaro, G. Invernici, T. Cenci, G. Maira, E. A. Parati, G. Stassi, L. M. Larocca and R. De Maria, Tumour Vascularization via Endothelial Differentiation of Glioblastoma Stem-like Cells, Nature, 2010, 468(7325), 824-828, DOI: 10.1038/ nature09557.

27 Y. Soda, T. Marumoto, D. Friedmann-Morvinski, M. Soda, F. Liu, H. Michiue, S. Pastorino, M. Yang, R. M. Hoffman, S. Kesari and I. M. Verma, Transdifferentiation of Glioblastoma Cells into Vascular Endothelial Cells, Proc. Natl. Acad. Sci. U. S. A., 2011, 108(11), 4274-4280, DOI: 10.1073/ pnas.1016030108.

28 R. Wang, K. Chadalavada, J. Wilshire, U. Kowalik, K. E. Hovinga, A. Geber, B. Fligelman, M. Leversha, C. Brennan and V. Tabar, Glioblastoma Stem-like Cells Give Rise to Tumour Endothelium, Nature, 2010, 468(7325), 829-833, DOI: 10.1038/nature09624.

29 M. Kapałczyńska, T. Kolenda, W. Przybyła, M. Zajączkowska, A. Teresiak, V. Filas, M. Ibbs, R. Bliźniak, Ł. Łuczewski and
K. Lamperska, 2D and 3D Cell Cultures - a Comparison of Different Types of Cancer Cell Cultures, aoms, 2016, 14(4), 910-919, DOI: 10.5114/aoms.2016.63743.

30 C. Jensen and Y. Teng, Is It Time to Start Transitioning From 2D to 3D Cell Culture?, Front. Mol. Biosci, 2020, 7, 33, DOI: $10.3389 /$ fmolb.2020.00033.

31 C. Wang, X. Chen, B. Wang, M. Huang, B. Wang, Y. Jiang and R. S. Ruoff, Freeze-Casting Produces a Graphene Oxide Aerogel with a Radial and Centrosymmetric Structure, ACS Nano, 2018, 12(6), 5816-5825, DOI: 10.1021/ acsnano.8b01747.

32 D. R. Dreyer, S. Park, C. W. Bielawski and R. S. Ruoff, The Chemistry of Graphene Oxide, Chem. Soc. Rev., 2010, 39(1), 228-240, DOI: 10.1039/B917103G.

33 L. Shahriary and A. A. Athawale, Graphene Oxide Synthesized by Using Modified Hummers Approach, Int. J. Energy Environ. Eng., 2014, 02(01), 58-63.

34 L. Ghasemi-Mobarakeh, M. P. Prabhakaran, M. Morshed, M. H. Nasr-Esfahani and S. Ramakrishna, Electrical Stimulation of Nerve Cells Using Conductive Nanofibrous Scaffolds for Nerve Tissue Engineering, Tissue Eng., Part A, 2009, 15(11), 3605-3619, DOI: 10.1089/ten.tea.2008.0689.

35 C. E. Schmidt, V. R. Shastri, J. P. Vacanti and R. Langer, Stimulation of Neurite Outgrowth Using an Electrically Conducting Polymer, Proc. Natl. Acad. Sci. U. S. A., 1997, 94(17), 8948-8953, DOI: 10.1073/pnas.94.17.8948.

36 Y. Wang and L. Guo, Nanomaterial-Enabled Neural Stimulation, Front. Neurosci., 2016, 10, DOI: 10.3389/ fnins.2016.00069.

37 Z. Zhang, M. Rouabhia, Z. Wang, C. Roberge, G. Shi, P. Roche, J. Li and L. H. Dao, Electrically Conductive Biodegradable Polymer Composite for Nerve Regeneration: Electricity-Stimulated Neurite Outgrowth and Axon Regeneration, Artif. Organs, 2007, 31(1), 13-22, DOI: 10.1111/ j.1525-1594.2007.00335.x.

38 A. J. Engler, S. Sen, H. L. Sweeney and D. E. Discher, Matrix Elasticity Directs Stem Cell Lineage Specification, Cell, 2006, 126(4), 677-689, DOI: 10.1016/j.cell.2006.06.044.

39 B. P. Chan and K. W. Leong, Scaffolding in Tissue Engineering: General Approaches and Tissue-Specific Considerations, Eur. Spine J., 2008, 17(S4), 467-479, DOI: 10.1007/ s00586-008-0745-3.

40 P. C. Georges, W. J. Miller, D. F. Meaney, E. S. Sawyer and P. A. Janmey, Matrices with Compliance Comparable to That of Brain Tissue Select Neuronal over Glial Growth in Mixed Cortical Cultures, Biophys. J., 2006, 90(8), 3012-3018, DOI: 10.1529/biophysj.105.073114.

41 J. M. Barnes, L. Przybyla and V. M. Weaver, Tissue Mechanics Regulate Brain Development, Homeostasis and Disease, J. Cell Sci., 2017, 130(1), 71-82, DOI: 10.1242/jcs.191742.

42 J. T. S. Pettikiriarachchi, C. L. Parish, M. S. Shoichet, J. S. Forsythe and D. R. Nisbet, Biomaterials for Brain Tissue Engineering, Aust. J. Chem., 2010, 63(8), 1143, DOI: 10.1071/ CH10159.

43 L. Krishna, K. Dhamodaran, C. Jayadev, K. Chatterjee, R. Shetty, S. S. Khora and D. Das, Nanostructured Scaffold 
as a Determinant of Stem Cell Fate, Stem Cell Res. Ther., 2016, 7(1), 188, DOI: 10.1186/s13287-016-0440-y.

44 S. K. Seidlits, Z. Z. Khaing, R. R. Petersen, J. D. Nickels, J. E. Vanscoy, J. B. Shear and C. E. Schmidt, The Effects of Hyaluronic Acid Hydrogels with Tunable Mechanical Properties on Neural Progenitor Cell Differentiation, Biomaterials, 2010, 31(14), 3930-3940, DOI: 10.1016/j.biomaterials.2010.01.125.

45 T.-W. Wang and M. Spector, Development of Hyaluronic AcidBased Scaffolds for Brain Tissue Engineering, Acta Biomater., 2009, 5(7), 2371-2384, DOI: 10.1016/j.actbio.2009.03.033.

46 S. R. Hynes, M. F. Rauch, J. P. Bertram and E. B. Lavik, A Library of Tunable Poly(Ethylene Glycol)/Poly(L-Lysine) Hydrogels to Investigate the Material Cues That Influence Neural Stem Cell Differentiation, J. Biomed. Mater. Res., 2009, 89A(2), 499-509, DOI: 10.1002/jbm.a.31987.

47 M. Büyüköz, E. Erdal and S. Alsoy Altinkaya, Nanofibrous Gelatine Scaffolds Integrated with Nerve Growth Factorloaded Alginate Microspheres for Brain Tissue Engineering, J. Tissue Eng. Regen. Med., 2018, 12(2), 707-719, DOI: 10.1002/term.2353.

48 A. Mostafavi, H. Daemi, S. Rajabi and H. Baharvand, Highly Tough and Ultrafast Self-Healable Dual Physically Crosslinked Sulfated Alginate-Based Polyurethane Elastomers for Vascular Tissue Engineering, Carbohydr. Polym., 2021, 257, 117632, DOI: 10.1016/j.carbpol.2021.117632.

49 J. A. W. van Dommelen, T. P. J. van der Sande, M. Hrapko and G. W. M. Peters, Mechanical Properties of Brain Tissue by Indentation: Interregional Variation, J. Mech. Behav. Biomed. Mater., 2010, 3(2), 158-166, DOI: 10.1016/j.jmbbm.2009.09.001.

50 X. Jin, F. Zhu, H. Mao, M. Shen and K. H. Yang, A Comprehensive Experimental Study on Material Properties of Human Brain Tissue, J. Biomech., 2013, 46(16), 2795-2801, DOI: 10.1016/j.jbiomech.2013.09.001.

51 S. Chatelin, J. Vappou, S. Roth, J. S. Raul and R. Willinger, Towards Child versus Adult Brain Mechanical Properties, J. Mech. Behav. Biomed. Mater., 2012, 6, 166-173, DOI: 10.1016/j.jmbbm.2011.09.013.

52 J. Weickenmeier, R. de Rooij, S. Budday, P. Steinmann, T. C. Ovaert and E. Kuhl, Brain Stiffness Increases with Myelin Content, Acta Biomater., 2016, 42, 265-272, DOI: 10.1016/j.actbio.2016.07.040.

53 K. Miller, K. Chinzei, G. Orssengo and P. Bednarz, Mechanical Properties of Brain Tissue In Vivo: Experiment and Computer Simulation, J. Biomech., 2000, 33(11), 1369-1376, DOI: 10.1016/S0021-9290(00)00120-2.

54 I. Bružauskaitè, D. Bironaitè, E. Bagdonas and E. Bernotienè, Scaffolds and Cells for Tissue Regeneration: Different Scaffold Pore Sizes-Different Cell Effects, Cytotechnology, 2016, 68(3), 355-369, DOI: 10.1007/s10616-015-9895-4.

55 J. M. Sobral, S. G. Caridade, R. A. Sousa, J. F. Mano and R. L. Reis, Three-Dimensional Plotted Scaffolds with
Controlled Pore Size Gradients: Effect of Scaffold Geometry on Mechanical Performance and Cell Seeding Efficiency, Acta Biomater., 2011, 7(3), 1009-1018, DOI: 10.1016/ j.actbio.2010.11.003.

56 C. M. Murphy, M. G. Haugh and F. J. O'Brien, The Effect of Mean Pore Size on Cell Attachment, Proliferation and Migration in Collagen-Glycosaminoglycan Scaffolds for Bone Tissue Engineering, Biomaterials, 2010, 31(3), 461-466, DOI: 10.1016/j.biomaterials.2009.09.063.

57 Q. Zhang, H. Lu, N. Kawazoe and G. Chen, Pore Size Effect of Collagen Scaffolds on Cartilage Regeneration, Acta Biomater., 2014, 10(5), 2005-2013, DOI: 10.1016/ j.actbio.2013.12.042.

58 N. Li, Q. Zhang, S. Gao, Q. Song, R. Huang, L. Wang, L. Liu, J. Dai, M. Tang and G. Cheng, Three-Dimensional Graphene Foam as a Biocompatible and Conductive Scaffold for Neural Stem Cells, Sci. Rep., 2013, 3(1), 1604, DOI: 10.1038/srep01604.

59 S. Ruijtenberg and S. van den Heuvel, Coordinating Cell Proliferation and Differentiation: Antagonism between Cell Cycle Regulators and Cell Type-Specific Gene Expression, Cell Cycle, 2016, 15(2), 196-212, DOI: 10.1080/ 15384101.2015.1120925.

60 D. D. Azorín and F. Winkler, Two Routes of Direct Intercellular Communication in Brain Cancer, Biochem. J., 2021, 478(6), 1283-1286, DOI: 10.1042/BCJ20200990.

61 G. Pinto, I. Saenz-de-Santa-Maria, P. Chastagner, E. Perthame, C. Delmas, C. Toulas, E. Moyal-JonathanCohen, C. Brou and C. Zurzolo, Patient-Derived Glioblastoma Stem Cells Transfer Mitochondria through Tunneling Nanotubes in Tumor Organoids, Biochem. J., 2021, 478(1), 21-39, DOI: 10.1042/BCJ20200710.

62 A. L. Parker, M. Kavallaris and J. A. McCarroll, Microtubules and their Role in Cellular Stress in Cancer, Front. Oncol., 2014, 4, 153, DOI: 10.3389/fonc.2014.00153.

63 L. Cirillo, M. Gotta and P. Meraldi, The Elephant in the Room: The Role of Microtubules in Cancer, in Cell Division Machinery and Disease, ed. M. Gotta and P. Meraldi, Advances in Experimental Medicine and Biology, Springer International Publishing, Cham, 2017, vol. 1002, pp. 93124, DOI: 10.1007/978-3-319-57127-0_5.

64 M. A. Jordan and L. Wilson, Microtubules as a Target for Anticancer Drugs, Nat. Rev. Cancer, 2004, 4(4), 253-265, DOI: $10.1038 / \mathrm{nrc1317.}$

65 L. E. Sidney, M. J. Branch, S. E. Dunphy, H. S. Dua and A. Hopkinson, Concise Review: Evidence for CD34 as a Common Marker for Diverse Progenitors, Stem Cells, 2014, 32(6), 1380-1389, DOI: 10.1002/stem.1661.

66 D. Ribatti and V. Djonov, Intussusceptive Microvascular Growth in Tumors, Cancer Lett., 2012, 316(2), 126-131, DOI: $10.1016 /$ j.canlet.2011.10.040. 